\title{
Anabases
}

ANABASES Traditions et réceptions de l'Antiquité

1 | 2005

Varia

\section{En guise de présentation}

\section{Pascal Payen}

\section{OpenEdition}

Journals

Édition électronique

URL : http://journals.openedition.org/anabases/1499

DOI : 10.4000/anabases.1499

ISSN : 2256-9421

\section{Éditeur}

E.R.A.S.M.E.

\section{Édition imprimée}

Date de publication : 1 mars 2005

Pagination : 277-283

ISSN : 1774-4296

\section{Référence électronique}

Pascal Payen, «En quise de présentation», Anabases [En ligne], 1 | 2005, mis en ligne le 01 octobre

2011, consulté le 20 octobre 2019. URL : http://journals.openedition.org/anabases/1499 ; DOI

10.4000/anabases.1499

Ce document a été généré automatiquement le 20 octobre 2019

(c) Anabases 


\section{En guise de présentation}

\section{Pascal Payen}

L'objet de ce «chantier historiographique » est de faire régulièrement le point sur les travaux dispersés qui traitent de la réception des historiens grecs et latins, à partir du $\mathrm{VI}^{\mathrm{e}}$ siècle de notre ère, en Orient et en Occident, jusqu'à leur implication dans les débats très contemporains qui abordent les questions et problèmes de l'écriture de l'histoire. Dans sa visée programmatique, la première livraison de cette chronique est une invitation adressée aux chercheurs à présenter d'autres dossiers, à formuler d'autres hypothèses - sur un auteur, un genre, un problème épistémologique, un ensemble de parutions, un projet éditorial en cours, un séminaire, les ressources d'un centre de recherche, ou encore les sites internet en ce domaine, etc. -, en privilégiant le mode de l'information et de l'échange. En bref, cette chronique est conçue comme un point de rencontre des publications et des initiatives, et comme un lieu de libre confrontation des hypothèses, sans exclusive ni orthodoxie.

La liste des problèmes qui seront abordés est ample; ceux qui sont mentionnés cidessous le sont simplement à titre d'invitation à prendre le relais dans les prochains numéros d'Anabases :

- Quelles fonctions sont assignées aux historiens anciens, dans l'éducation et la culture, à partir de leur redécouverte au $\mathrm{XV}^{\mathrm{e}}$ siècle ?

- Historiens anciens et « Antiquaires »

- L'historiographie ancienne : source, document ou archive?

- Connaissance et statut des historiens anciens à travers les Histoires anciennes

- Composition et histoire des corpus de fragments : Fragmenta Historicorum Graecorum (Müller), Fragmente der griechischen Historiker (Jacoby), et Historicorum Romanorum Reliquiae (Peter).

- Éditions et traductions, à partir du $\mathrm{XV}^{\mathrm{e}}$ siècle: le paratexte et les outils de l'interprétation

- L'étude des historiens anciens, entre histoire scientifique et histoire des représentations (XIX et $\mathrm{XX}^{\mathrm{e}}$ siècle).

Pour tenir leur rôle d'outil de la recherche, les réflexions qui suivent mentionnent également quelques-uns des travaux importants parus depuis quinze ans, mais sans se limiter à cette date. Plutôt que livrer les références en une liste finale, il a paru 
préférable de les insérer, de manière plus problématique, dans le fil de l'exposé et de les placer en notes.

\section{Prolégomènes}

1 Jusqu'à la fin du XVIII ${ }^{e}$ siècle et au début du XIX ${ }^{e}$, les historiens de l'Antiquité servent de modèles rhétoriques et de sources aux Modernes qui écrivent, en Europe, des Histoires anciennes de la Grèce, de Rome et des "nations barbares ». La connaissance érudite des œuvres d'Hérodote et de Tite-Live, de Thucydide et de Tacite, mais aussi de Polybe, Diodore, Denys d'Halicarnasse conduit à l'écriture de vastes recueils qui, à l'image de l'Histoire ancienne de Charles Rollin ${ }^{18}$,ou de l'History of Ancient Greece de John Gillies $^{19}$, sont un hommage rendu à l'art de la narration historique conçue par les Anciens. Recopiés, démarqués, plagiés, ces derniers ont longtemps tenu lieu de palimpsestes, jusqu'à ce que leur statut de «monument " s'efface en partie et soit remplacé, dans les bouleversements qui affectent l'atelier de l'historien tout au long du XIX siècle, par celui de « document $»^{20}$.

Or cette mutation épistémologique est étroitement associée aux changements dans les rapports que nous entretenons avec l'Antiquité, à la fois comme passé et comme repère pour comprendre le présent. C'est en effet dans le temps même où les historiens anciens servent de sources principales, entre le $\mathrm{XV}^{\mathrm{e}}$ et le XVIII ${ }^{\mathrm{e}}$ siècles ${ }^{21}$, que se forgent les «images» de l'Antiquité dont nous sommes souvent encore tributaires ${ }^{22}$. Par la suite, lorsque l'histoire se construit comme science, grâce à des travaux comme ceux de Louis de Beaufort (1703-1795) ${ }^{23}$, de Christian Gottlob Heyne (1729-1812), dans son séminaire de l'université de Göttingen, ou de Edward Gibbon (1737-1794), puis dans l'université prussienne, c'est l'Antiquité qui sert de laboratoire. La "science de l'Antiquité » - Altertumswissenschaft : le terme est forgé par Wolf, en 1806-1807 - sert d'arme à la Prusse, au moment où elle mène ses "guerres de libération " contre l'envahisseur napoléonien. L'université de Berlin est fondée dès 1810, à l'initiative de Wilhelm von Humboldt (1767-1835), qui s'appuie sur des antiquisants et des philologues tels que Friedrich August Wolf (1759-1824) et Barthold Georg Niebuhr (1776-1831). Wolf, l'auteur des Prolegomena ad Homerum, en 1795, exhorte publiquement les Allemands à défendre les études classiques comme un palladium de la patrie contre «des mains impies $»^{24}$. Pour Niebuhr, l'organisation des études philologiques doit être une des tâches essentielles du gouvernement prussien. Dans le rapport de force entre les sources, les historiens anciens exercent une influence déterminante, à l'image de Thucydide, qui sera très vite érigé en modèle d'historien scientifique, parce qu'épris d'« exactitude » et de « vérité ».

\section{La réception des historiens anciens : un champ d'étude à construire}

3 L'objet principal de cette chronique est de présenter un domaine de recherche: le problème de la réception des historiens anciens, qu'il est possible de ramener, provisoirement du moins, à deux questions principales. D'une part, étant donné qu'ils ont été pendant longtemps des documents privilégiés, lus de surcroît comme des monuments, quelles influences ont-ils exercées - par différence avec les autres sources - sur la construction de l'image que nous avons des sociétés anciennes? D’autre part, 
comment ont-ils contribué, en tant qu'historiens, ou plutôt parce qu'ils sont reconnus comme tels, à délimiter les contours du champ historique, compris dans son acception la plus large, et à préciser les règles de l'écriture de l'histoire ? Dans le temps long qui conduit des premières tentatives pour réfléchir sur le passé de la Grèce et de Rome dans ses rapports avec le présent - que l'on place ces débuts dès l'Antiquité ellemême ${ }^{25}$, ou bien avec Augustin, ou encore à Byzance ${ }^{26}$ - jusqu'aux ambitions d'une histoire scientifique, qui prend forme en Europe dans les années $1820-1830^{27}$ et qui constitue toujours, peu ou prou, notre cadre de travail, les historiens anciens, avec leurs questions, leurs récits, leurs manières de s'arrimer à leurs prédécesseurs pour, tout à la fois, les contester et former avec eux une tradition, ont toujours tenu une place de premier plan, dont l'étude reste à faire, dans une perspective d'archéologie du savoir et du discours sur l'Antiquité, et à l'horizon de la formation de la science historique contemporaine. Certes, il existe des analyses sur un auteur et sur une période limitée ${ }^{28}$, sur une tradition ${ }^{29}$, sur l'historiographie des études en rapport avec l'Antiquité ${ }^{30}$, mais non sur la réception même du corpus des historiens anciens, dans son ensemble, pour la fabrique de l'histoire. Il n'est pas, ou fort peu, tenu compte de leur influence dans la constitution des disciplines qui se rattachent à l'enquête historique, dans la genèse des genres d'écriture qui en découlent, dans l'émergence des formes de conscience historique associées aux luttes politiques ${ }^{31}$, alors même que les milieux, savants ou non, qui contribuent à ces activités sont formés de lecteurs des historiens anciens ou sont eux-mêmes des antiquisants. Dans ces milieux, quelle importance ont eue les éditions, les traductions disponibles, ou, à l'inverse, quelle incidence a eue leur quasi absence, comme dans le cas d'Hérodote, en France, entre 1645 et 1786 ? En quoi La Guerre du Péloponnèse de Thucydide contribue-t-elle, parmi d'autres facteurs, à la formation du modèle athénien et aux prémices du discrédit qui affecte la Sparte idéalisée, dès les années 1730-1760 ? Comment la vision propre d'un historien ancien est-elle projetée par les Modernes sur la période qu'il étudie : ainsi de Tacite, historien sombre d'une période qui ne peut être que de turpitudes?

Les historiens anciens sont le plus souvent considérés comme de lointains précurseurs de leurs " collègues » modernes, sur le plan de la méthode et de l'écriture, les seconds s'inscrivant dans la ligne d'un progrès et d'un perfectionnement, en dépit de la révérence due à une origine prestigieuse. Comment faire plus rigoureux que Thucydide, plus amèrement lucide que Tacite dans l'analyse des méandres du pouvoir, plus "synoptique » que Polybe dans l'appréciation globale d'événements éloignés dans l'espace? La perspective que nous proposons ici est inverse ; elle consiste à repérer comment, par un ensemble d'emprunts, mais surtout d'écarts, de ruptures, parfois de méprises, l'épistémologie et l'écriture de l'histoire des Modernes se sont façonnées à partir des pratiques des Anciens, imités ou contestés.

\section{La redécouverte de Thucydide comme historien politique}

5 Un des dossiers parmi les plus prometteurs, pour illustrer les enjeux de la réception des historiens anciens, est celui des raisons de l'oubli de Thucydide, à partir des années 1650 (la dernière grande traduction, en anglais, due à Hobbes, est publiée à Londres, en 1648), et de sa progressive redécouverte au XVIII ${ }^{e}$ siècle, couronnée par la traduction et le commentaire de Pierre-Charles Lévesque, en 1795. Pourquoi le XVIII siècle, 
notamment en France, a-t-il laissé La Guerre du Péloponnèse dans l'oubli ? Pourquoi Thucydide a-t-il été « la grande victime des Lumières »" ?

6 Un tel phénomène s'inscrit certainement à l'intérieur du mouvement d'ensemble que l'on a déjà signalé, mais il doit aussi avoir ses raisons propres, car, à la différence de la plupart des autres historiens grecs, l'oubli qui affecte Thucydide se situe entre deux apogées. Dans l'Antiquité, et depuis le IV siècle avant notre ère, il est le modèle de l'«histoire véritable »33, celui dont les continuateurs - Xénophon, Théopompe, Philistos, Cratippos - se pressent, et que plusieurs traités proposent à l'imitation: Praxiphane, un élève de Théophraste, à la fin du IV siècle, est en ce sens le précurseur de Lucien, pour qui, à la manière de Thucydide, «l'unique tâche de l'historien est de raconter les faits comme ils se sont passés ( $\dot{\omega} \zeta \dot{\varepsilon} \pi \rho \alpha ́ x \theta \eta) »^{34}$. Cette tradition passe par Éphore, Polybe, Salluste, Flavius Josèphe, Dion Cassius, et domine encore l'historiographie byzantine. Dans la notice que Photios, au IXe siècle, consacre à Hérodote, ce sont les catégories de l'historiographie thucydidéenne qui prévalent et qui

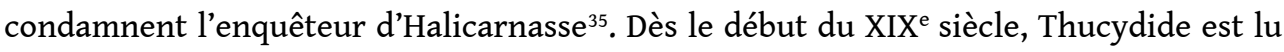
avec beaucoup d'attention, en tant qu'historien politique, par les fondateurs de l'Université de Berlin, en particulier par Niebuhr, qui lui-même influença Ranke et l'élève de ce dernier, Wilhelm Roscher, auteur d'un livre capital: Leben, Werk und Zeitalter des Thukydides (Göttingen, 1842). Pour ces savants - et il en est ainsi jusqu'à Eduard Meyer, qui synthétise ce courant interprétatif dans une monographie publiée en $1913^{36}$ - Thucydide est l'historien de l'Athènes de Périclès, épris de vérité, de rigueur et de respect des sources, fondateur d'une histoire contemporaine, avant tout politique et militaire, qui éclaire les enjeux du présent.

7 Entre ces deux apogées, comment rendre compte d'un oubli surprenant? La traduction latine de Thucydide par Lorenzo Valla est en effet publiée dès 1483 et réimprimée dans toute l'Europe au cours du XVI ${ }^{e}$ siècle ; l'édition aldine parait en 1502 ; Casaubon traduit en latin la Vie de Thucydide de Marcellinos, qui accompagne la réédition de la traduction de Valla, chez Henri Estienne, en 1589. Trois hypothèses peuvent être suggérées pour expliquer l'absence de Thucydide quelques décennies plus tard. Tout d'abord, la réflexion politique de Thucydide pouvait difficilement prendre place à l'intérieur du cadre conceptuel de l'histoire humaniste. En effet, s'il est considéré comme «le plus fidèle et le plus sincère des historiens $\aleph^{37}$, c'est pour des raisons non politiques, mais morales, et celles-ci se retournent contre lui lorsqu'elles sont appliquées à son style, considéré comme trop austère et comme obscur à force de concision ${ }^{38}$ Ensuite, la puissance d'attraction du modèle spartiate, tel qu'il figure chez Rousseau et Mably, ainsi que dans l'Encyclopédie ${ }^{39}$, a sûrement contribué à laisser dans l'ombre la dimension politique - sur les plans extérieur et intérieur - du conflit dans lequel sont engagées Athènes et les cités grecques. C'est en effet sur la morale, telle qu'on peut l'observer dans une Sparte idéalisée, que pourra être reconstruite la politique, et non l'inverse. ${ }^{40}$ Enfin, il est permis de se demander si l'Antiquité imaginaire, riche en modèles pour les lecteurs de Plutarque, ou bien construite de toutes pièces pour dénoncer la corruption du monde moderne, à partir de la Sparte de Lycurgue et de la Rome républicaine, ne fait pas écran, pour l'heure, à une histoire dont les données "positives ", en matière politique et militaire, seront bientôt reprises par ceux qui sont préoccupés de lutter précisément contre l'influence et la présence françaises, sur leur sol et en Europe, ou bien par les tenants de la pensée libérale, en Angleterre et en France, tels que Grote et Duruy. 
8 Le mouvement inverse, de redécouverte, s'amorce, semble-t-il, lorsque la réflexion en matière politique se détache de la personne du prince et porte sur les institutions. En dépit des divergences entre Thucydide et Rollin, il n'est pas sans conséquence, pour la réception nouvelle de l'Athénien, de remarquer que Rollin le qualifie d'« excellent historien $»^{41}$, en raison de la sûreté de ses informations, qui lui ont permis, notamment au livre 5 de son Histoire ancienne, de mener la comparaison entre Sparte et Athènes, en faveur de celle-ci. La lecture de Thucydide aurait-elle pu contribuer à la maturation de L'Esprit des Lois (1748) ${ }^{42}$ ? La reconnaissance de la dimension politique de l'expérience grecque, telle qu'elle apparaît dans les luttes entre cités et à travers leurs institutions, est un phénomène caractéristique des années 1730-1760, qui n'a pas encore été pleinement étudié. L'analyser à partir de la réception et de la redécouverte de l'œuvre de Thucydide - il faudrait tenir compte aussi de la réception de Polybe - permettrait certainement de mettre aussi au jour les prémices d'un changement de paradigme: l'effacement du modèle spartiate et l'émergence de l'histoire et de la référence athéniennes. Dans ce chapitre de l'histoire de l'historiographie, la place de Thucydide a été, jusqu'ici, trop négligée. Conduire cette enquête permettrait, de surcroît, de mieux comprendre les choix de Pierre-Charles Lévesque, dans sa traduction et son commentaire de 1795, en faveur d'un Thucydide historien du politique. Dès sa préface, il donne le ton, en affirmant qu'«il doit être le plus étudié dans les pays où tous les citoyens peuvent avoir un jour quelque part au gouvernement», et, ajoute-t-il peu après: «un membre très éclairé du Parlement d'Angleterre disait qu'il ne pouvait s'agiter dans les Chambres aucune question sur laquelle on ne trouvât des lumières dans Thucydide $»^{43}$.

\section{NOTES}

18. Ch. Rolun, Histoire ancienne des Égyptiens, des Carthaginois, des Assyriens, des Mèdes et des Perses, des Macédoniens, des Grecs, Paris, Vve Etienne, 13 vol., 1731-1738.

19. J. GILLIES, The History of Ancient Greece, its colonies and conquests, from the earliest accounts till the division of the Macedonian empire in the East including the history of literature, philosophy and the fine arts, London, A. Strahan, 2 vol., 1786. L'ouvrage est aussitôt traduit en français: Histoire de l'ancienne Grèce..., Paris, Buisson, 6 vol., 1787-1788.

20. Cf. M. Foucault, L'archéologie du savoir, Paris, Gallimard, 1969, p. 13-15.

21. Cf. J.-M. Dufays et Ch. Grell (éd.), Pratiques et concepts de l'histoire en Europe, XVI ${ }^{e}-X V I I I^{e}$ siècles, Paris, Publications de la Sorbonne, 1990 ; Ch. GRELL, L'histoire entre érudition et philosophie. Essai sur la connaissance historique à l'âge des Lumières, Paris, P.U.F., 1993, p. 125-191.

22. Jusqu'aux années 1810-1820, ni l'archéologie, ni les inscriptions, ni les œuvres d'art n'apportent une contribution systématique à l'analyse historique, en l'absence des grands corpus documen-taires.

23. Louis de BEAUFORT, Dissertation sur l'incertitude des cinq premiers siècles de l'histoire romaine, Utrecht, E. Neaulme, 1738.

24. Cité par B. Bravo, Philologie, histoire, philosophie de l'histoire. Étude sur J.G. Droysen historien de l'Antiquité, Varsovie, 1968, rééd. Hildesheim-Zurich-New York, Georg Olms Verlag, 1988, p. 67-68. 
25. Cf. F. HARTOG, "La storiografia fra passato e presente" in S. SETtis (a cura di), I Greci. Storia, Cultura, Arte, Società, 2. Una storia greca, II. Definizione, Turin, Einaudi, 1997, p. 960-961, et L'Histoire, d'Homère à Augustin, Préfaces des historiens et textes sur l'histoire, réunis et commentés par F. HARTOG, traduits par M. CASEVITZ, Paris, Seuil, 1999.

26. Les études de P. LEMERLE réunies dans Le premier humanisme byzantin. Notes sur enseignement et culture à Byzance des origines au Xème siècle, Paris, P.U.F., 1971, contiennent de nombreuses données sur les historiens, de même que, pour la période postérieure, le livre de N. G. WiLson, From Byzantium to Italy. Greek Studies in the Italian Renaissance, London, Duckworth, 1992.

27. La synthèse de A. Momigliano, “Les origines des recherches sur l'Antiquité ", in Les fondations du savoir historique, Paris, Les Belles Lettres, 1992, p. 61-91 (éd. originale The Classical Foundations of Modern Historiography, Berkeley..., University of California Press, 1990) traite de l'influence des historiens anciens jusqu'à Max Weber, mais de manière non systématique.

28. Cf. C. V OlpILHAC-Auger, Tacite en France de Montesquieu à Chateaubriand, Oxford, Voltaire Foundation ( $n^{\circ}$ 313), 1993 ; C. Montepaone, G. IMbruglia, M. CATARZI, M.L. SilveStre (a cura di), Tucidide nelle storiografia moderna, Naples, Morano Editorie, 1994. Le pionnier en ce domaine est encore A. Momigliano : cf. par exemple “Polybius' Reappearance in Western Europe" (1974), trad. fr.: "La redécouverte de Polybe en Europe occidentale", dans Problèmes d'historiographie ancienne et moderne, Paris, Gallimard, 1983, p. 186-209 (avec les références concernant les éditions de cette étude, p. 186) ; “The First Political Commentary on Tacitus" (1947), trad. fr.: "Le premier commentaire politique de Tacite ", Ibid., p. 210-243 (références, p. 210), et “ Tacite et la tradition tacitéenne ", in Fondations, p. 127-153.

29. Cf. C. Ampolo, Storie greche. La formazione della moderna storiografia sugli antichi Greci, Turin, Einaudi, 1997. Pour l'histoire romaine, cf. les références indiquées par C. Ampolo, p. XIV, n. 1.

30. On renverra seulement à la synthèse classique de R. PFEIFFER, History of Classical Scholarship. From 1300 to 1850, Oxford, At the Clarendon Press, 1976. C'est une large part de l'œuvre de Momigliano qu'il faudrait encore citer.

31. Fait exception l'étude de B. B oudou, “La réception d'Hérodote au XVI siècle ", in G. LACHENAUD et D. LONGRÉE (dir.), Grecs et Romains aux prises avec l'histoire. Représentations, récits et idéologie, Rennes, P. U. de Rennes, 2003, p. 729-743.

32. Cf. Ch. GReLl, Le Dix-huitième et l'Antiquité en France, 1680-1789, Oxford, Voltaire Foundation, 1995, p. 137.

33. Cf. A. Momigliano, "Les traditions hérodotéenne et thucydidéenne", in Fondations, p. 50-55, ainsi que S. HORNBLOWER, “The Fourth-century and Hellenistic Reception of Thucydides”, Journal of Hellenic Studies 115, 2 (1995), p. 47-68.

34. Lucien, Comment il faut écrire l'histoire 39 (ainsi que 42, 47, 49, 51), à rapprocher de Thucydide II, $48,3$.

35. Photios, Bibliothèque 60, $19 \mathrm{~b}$ 16-42.

36. E. MEYER, Thukydides und die Entstehung der Wissenschaftlichen Geschichtsschreibung, Vienne et Leipzig, 1913. Sur la tradition thucydidéenne, de Niebuhr à Meyer, cf. C. MonTEPAONE, G. IMBRUGLIA, M. CATARzi, M.L. Silvestre, Tucidide. Ce courant dépasse largement les frontières de l'Allemagne : cf. J. de Romilly, Thucydide et l'impérialisme athénien, Paris, Les Belles Lettres, 1947, et Histoire et raison chez Thucydide, Paris, Les Belles Lettres, 1956 ; J.H. FinLEY, Thucydides, Cambridge, Harvard University Press, 1942. Thucydide est jaugé et analysé à l'étalon du positivisme tout au long du commentaire de A.W. GOMME, A. ANDREWES et K.J. DOVER, A Historical Commentay on Thucydides, Oxford, Clarendon Press, 5 vol., 1945-1981; voir à ce sujet la distance prise par S. HORNBLOWER, A Commentary on Thucydides, Oxford, Clarendon Press, vol. II (Books IV-V. 24), 1996, p. 1-19 ("General remarks; the relation of this commentary to HCT").

37. Selon le P. RAPIN, La comparaison de Thucydide et de Tite-Live avec un jugement des défauts et des beautés de leurs ouvrages, Paris, 1681, p. 224. 
38. Ibid., p. 199. Sur la concision du style de Thucydide, cf. Cicéron, De Oratore II, 56.

39. L'article "Athènes ", absent dans la première édition, n'apparaît que dans le Supplément, I, p. 669-676, sous la plume de François Henri Turpin, et est très critique, alors que l'entrée “Lacédémone ", due à Jaucourt (t. IX, p. 152-160), développe les thèses de Rousseau.

40. Cf. JEAucourt, "Lacédémone", Encyclopédie: “C'est là que, par une morale purement naturelle, on voyait des hommes assujettis à la raison, qui, par leur propre choix, se rangeaient sous une austère discipline, et qui, soumettant les autres peuples à la force des armes, se soumettaient eux-mêmes à la vertu : un seul Lycurgue leur en traça le chemin [...] Lycurgue me tient lieu de toutes choses ; plus de Solon ni d'Athènes ".

41. Ch. Rollin, Histoire ancienne, livre 27, § “ Des historiens ”, p. 214 (éd. Letronne, 1821-1825).

42. La même question pourrait être posée à propos de Polybe pour les analyses concernant la problématique des “causes" et la dimension politique du déclin de Rome, dans les Considérations sur les causes de la grandeur des Romains et de leur décadence : l'ouvrage paraît en 1734, et sa seconde édition, revue et corrigée, en 1748, la même année que L'Esprit des Lois.

43. P.-Ch. LÉVESQue, Histoire de Thucydide, fils d'Olorus, Paris, J.B. Gail, an IV-1795, t. I, Préface, p. XXVII.

\section{AUTEUR}

PASCAL PAYEN

payen@univ-tlse2.fr 